\title{
A rare fatal case of internal hernia caused by Meckel's diverticulum in a paediatric patient
}

\author{
Vandana Jain, Sanjay Sahi
}

Queen Mary’s Hospital, Department of Paediatrics, Sidcup, UK. E-mail: jainvan@hotmail.com

Received 26 April 2011; revised 25 May 2011; accepted 2 June 2011.

\begin{abstract}
We describe a very rare case of an internal hernia associated with a Meckel's diverticulum, which lead to the death of a young 3 year-old boy. The case describes symptoms of abdominal pain and vomiting, on a background of previous intermittent abdominal pain. The possibility of small bowel obstruction was suspected, and appropriate imaging was performed. This case illustrates the need for a high index of suspicion for small bowel obstruction, with appropriate investigations and review. It also highlights the limitations of imaging modalities in identifying complications of Meckel's diverticulum. It is important to raise awareness of this fatal cause for small bowel obstruction and to help identify suggestive imaging features, which may point towards a possible complicated Meckel's diverticulum. Earlier recognition and diagnosis could reduce morbidity and mortality.
\end{abstract}

Keywords: Meckel's Diverticulum; Internal Hernia; Small Bowel Obstruction

\section{INTRODUCTION}

Meckel's diverticulum (MD) is an embryonic remnant of the omphalomesenteric (vitelline) duct, which normally disappears by the seventh week of gestation [1]. It is the most common congenital anomaly of the gastrointestinal tract, occurring in $2 \%-3 \%$ of the population [2]. MD is a true diverticulum, containing all layers of the ileal wall, where heterotrophic tissue is present. Gastric mucosa is the most common heterotrophic tissue, followed by pancreatic tissue, then mixed pancreatic tissue and gastric mucosa [1]. It occurs in approximately equal frequency in both sexes, but symptoms more commonly present in males [3]. The most symptomatic age group is the under-2's. Most MDs are clinically silent and are often an incidental finding at laparotomy. Symptoms arise when there are associated complications, which include, haem- orrhage, small bowel obstruction (SBO) and diverticulitis.

We describe a rare case of SBO originating from herniation into the orifice formed from a congenital band from the diverticulum to the root of a mesentery. We aim to highlight the importance of this condition and its potential fatal consequence, as well as exploring reasons as to why diagnosis can be difficult.

\section{CASE REPORT}

A 3 year-old boy attended Paediatric Accident and Emergency, with an acute history of vomiting and abdominal pain. He looked unwell and had generalised abdominal tenderness, but no abdominal distension and normal bowel sounds. He was haemo-dynamically stable.

He had presented with a similar episode, 10 weeks previously, with a history of diarrhoea and vomiting, after a short holiday to Turkey. At this stage, he was also noticed to have abdominal distension. An abdominal x-ray revealed dilated large bowel loops, thought to be secondary to colitis or ileus. An abdominal computed tomography scan showed no abdominal obstruction. Blood, stool and urine tests were normal. The child recovered with intravenous fluids, and was discharged home after 48 hours with outpatient follow up arranged. The most likely diagnosis was gastroenteritis. Parents reported that he did have a past history of intermittent abdominal pain and vomiting, but no previous medical consultation.

During the current admission, an infective cause for his symptoms seemed most likely, and he was managed with intravenous fluids. Blood tests were normal, including inflammatory markers. A clean catch urine sample was awaited. Within 12 hours of admission, his vomiting settling, however the abdominal pain persisted. Hence, an abdominal ultrasound was performed. Visualization was difficult due to gas and tenderness, but upper abdominal viscera was normal and there was no evidence of malrotation or intussusception. He was otherwise well in himself with normal vital signs. A CT scan had been arranged for the next day for further in- 
vestigation of the persistent pain.

However, at 24 hours into the admission, the patient acutely deteriorated. He was showing signs of hypovolaemic shock, followed by a large dark brown vomit and a rigid abdomen, very suggestive of peritonitis. He quickly became unresponsive, and cardiopulmonary resuscitation was commenced, but unsuccessful.

Post-mortem diagnosis revealed haemorrhagic infarction of $47 \mathrm{~cm}$ of small bowel, which had herniated through a small gap in the mesentery, formed from a band running from a Meckel's diverticulum to the root of a mesentery.

\section{DISCUSSION}

Symptoms of abdominal pain and vomiting are common presentations to Paediatric Accident and Emergency. The majority of cases are viral infections, but a high index of suspicion for possible SBO is required. Any unusual features in the presentation, such as bilious vomiting, abdominal distension, abnormal bowel sounds, tympanic percussion, constipation, previous history of abdominal pain and vomiting, worsening or non-resolution of symptoms should ring alarm bells [4]. Although the presence of diarrhoea is unusual in small bowel obstruction, it does not exclude it. Our index case demonstrates that despite the physician's vigilence and high index of suspicion, this unusual cause of SBO is difficult to identify. We aim to raise awareness of this rare condition and it's diagnosis, to help reduce morbidity and mortality.

Meckel's Diverticula are usually asymptomatic. The most common complication to arise is haemorrhage. Other complications include diverticulitis and SBO. The mechanisms for SBO can involve a volvulus, intussusception, diverticulitis, foreign body impaction, inversion of the diverticulum into bowel lumen or hernia, neoplastic obstruction, or, as in our case, formation of an internal hernia [1]. An internal hernia is a particularly rare form of MD obstruction, and is caused by the MD attaching to the adjacent mesentery, forming an orifice for ileal loop herniation [2,6-8].

A few other case reports of MD associated internal hernia have been identified, with diagnosis at laparotomy for clinical and radiological evidence for SBO [6,7]. In our case, diagnosis of SBO was further complicated by initial resolution of symptoms and inconclusive imaging result.

We know that radiological signs of SBO are well established, but recognizing MD as the cause is very difficult pre-operatively. Some radiological features, which may point towards MD, in the setting of intestinal obstruction, have been suggested, but are not without their limitations [5].

Abdominal radiographs may be useful to identify MD by the presence of enteroliths [5], which can be seen as peripheral calcification with a radiolucent centre, often located in the right lower quadrant of the abdomen. However, as enteroliths are often absent, this sign will not always be illicited.

Ultrasonograms may suggest MD if there is evidence of a round or tubular cystic lesion, however, this can often mimic a dilated bowel loop or duplication cyst, and more commonly visualization is difficult due to overlying bowel loops $[1,6]$.

The use of barium studies is limited, due to poor barium filling of the obstructed bowel and their time consuming nature [9]. However, they can show MD as a blind-ended saccular or tubular lesion on the antimesenteric site of the ileum or as a triradiate fold pattern at the junction of the diverticulum and the ileum $[4,5]$.

Computed tomography (CT) is usually the gold standard imaging modality for intestinal obstruction, however, identifying MD as the cause can be extremely difficult [5,9]. It looks like a bowel loop and can be obscured by co-existant complications, such as intussussecption and volvulus [5,6]. If seen, it appears as a blind ending, tubular or sausage shaped structure that communicates with the small intestine lumen at the level of the obstruction. It may contain air, fluid, fecal matter or enteroliths. In particular, with an internal hernia, such as in our case, clustering of bowel loops and stretched crowded and engorged mesenteric vessels may be suggestive features [10].

A technique known as multidetector computed tomographic scan (MDCT) with high spatial resolution and isotropic multiplanar reconstruction images have demonstrated MD included in hernias [6,11,12], and is being used second line after the abdominal x-ray in suspected cases [6].

The technetium-99 m pertechnetate scan in children has a sensitivity of $80 \%-90 \%$, a specificity of $95 \%$ and an accuracy of $90 \%$. It is specific to ectopic gastric mucosa, hence may give false positives in gut duplication cysts with ectopic gastric mucosa [13]. Also false negative scans may be due to the rapid dilution of radioactive secondary to impaired vascular supply or insufficient gastric mucosa. However, if there is a complicated MD from clinical or radiological suggestion, the Meckel's scan is the most accurate non-invasive investigation to perform.

We conclude that in unclear diagnoses of abdominal pain and vomiting in young children, the uncommon but potentially catastrophic complications of MD associated obstruction should be considered, which may be difficult to diagnose radiologically. Awareness of the specific radiological features of the MD is important, although there are many limitations.

\section{REFERENCES}

[1] Debartolo, H.M. and Van Heerden, J.A. (1976) Meckel's diverticulum. Annals of Surgery, 183, 30-33. doi:10.1097/00000658-197601000-00006 
[2] Hawkins, H.B., Slavin, J.D. Jr, Levin, R. and Spencer, R.P. (1976) Meckel's diverticulum internal hernia and adhesions without gastrointestinal bleeding-ultrasound and scintographic findings. Clinical Nuclear Medicine, 21, 938-940. doi:10.1097/00003072-199612000-00004

[3] Chen, D.F., Tai, F.C. and, Chen, T.Y. (1990) Internal herniation caused by Meckel's diverticulum. J Surg Assoc ROC, 23, 184-186.

[4] McCollough, M. and Sharieff, G.Q. (2003) Abdominal surgical emergencies in infants and young children. Emergency Medicine Clinics of North America, 21, 909-935.

[5] Levy, A.D. and Hobbs, C.M. (2004) From the archives of the AFIP. Meckel diverticulum: Radiologic features with pathologic correlation. Radiographics, 24, 565-587.

[6] Ko, S.F., Tiao, M.M., Huang, F.C., Hsieh, C.S., Huang, C.C., Ng, S.H., Wan, Y.L. and Lee, T.Y. (2008) Internal hernia associated with Meckel's diverticulum in 2 pediatric patients. The American Journal of Emergency Medicine, 26, 86-90.

[7] Lee, M.C., Lin, L.H. and Chen, D.F. (2008) Internal hernia caused by Meckel diverticulum in an infant:
Report of one case. Acta Paediatrica Taiwanica, 42, 105.

[8] Murakami, R., Sugizaki, K., Kobayashi, Y., Ogura, J., Yamamoto, K., Kurokawa, A. and Kumazaki, T. (2008) Strangulation of small bowel due to Meckel diverticulum: CT findings. Clinical Imaging, 23, 181-183.

[9] Balthazar, E.J. (1994) CT of small bowel obstruction. American Journal of Roentgenology, 162, 255-261.

[10] Blachar, A., Federle, M.P., Dodson, S.F. (2001) Internal hernia: Clinical and imaging findings in 17 patients with emphasis on CT criteria. Radiology, 218, 68-74.

[11] Sinha, R. (2005) Bowel obstruction due to Littre hernia: CT diagnosis. Abdominal Imaging, 30, 682-684. doi:10.1007/s00261-005-0318-4

[12] Aggarwal, B.K., Rajan, S., Aggarwal, A., Gothi, R. and Tandon, V. (2005) CT diagnosis of Meckel's diverticulum in a paracolic internal hernia. Abdominal Imaging, 30, 56-59.

[13] Berquist, T.H., Nalan, N.G., Stephens D.H. and Carlson, H.C. (1976) 99mTc-perctechnetate in scintographic diagnosis of Meckel's diverticulum: Review of 100 cases. Journal of Nuclear Medicine, 17, 465-469. 